\title{
Near-Infrared Photoimmunotherapy for Cancers of the Gastrointestinal Tract
}

\author{
Tadanobu Nagaya $^{a, b}$ Peter L. Choyke ${ }^{a}$ Hisataka Kobayashi ${ }^{a}$ \\ aMolecular Imaging Program, Center for Cancer Research, National Cancer Institute, National Institutes of Health, \\ Bethesda, MD, USA; ${ }^{b}$ Department of Gastroenterology, Shinshu University Hospital, Matsumoto, Japan
}

\section{Keywords}

Near-infrared photoimmunotherapy · Digestive cancer ·

Fiber optic diffuser · Monoclonal antibodies · Endoscopy

\begin{abstract}
Background: Cancers of the gastrointestinal (Gl) tract are the common leading cause of cancer-related death in the world. Recent advances in cancer therapies such as intensive multidrug chemotherapy and molecular targeted treatment have improved therapeutic efficacy; however, the outcomes are not satisfied. Moreover, these therapies also cause severe side effects. New type of cancer therapies is urgently needed to improve the outcomes and to reduce side effects of GI tract cancers. Summary: This account is a comprehensive review article on the newly developed, photochemistry-based cancer therapy named as near-infrared photoimmunotherapy (NIR-PIT). NIR-PIT is a highly selective tumor treatment that employs an antibody-photoabsorber conjugate, which is activated by near-infrared light. A world-wide phase 3 clinical trial of NIR-PIT against recurrent head and neck cancer patients is currently underway. NIR-PIT differs from conventional cancer therapies such as surgery, chemotherapy, and radiation in its selectivity for killing cancer cells and cells treated with NIR-PIT leading to immunogenic cell death. Preclinical research in animals with combining cancer-targeting
\end{abstract}

NIR-PIT and other cancer immunotherapies could lead to responses not only in local tumor but also in distant metastases. NIR-PIT also leads to an immediate and dramatic increase in vascular permeability after therapy. From these aspects, NIR-PIT appears to be a promising new form of cancer therapy. NIR-PIT could be readily translated into clinical use for virtually any cancers in the near future provided suitable humanized antibodies are available. Here, we describe the specific advantages and applications of NIR-PIT in the GI tract. Key Messages: We believe that NIR-PIT with NIR excitation light, which can be delivered via a fiber optic diffuser through endoscopes, is a promising method for a new treatment of $\mathrm{Gl}$ cancers.

(c) 2020 S. Karger AG, Basel

\section{Introduction}

Despite rapid advances in diagnostic and therapeutic procedures, cancer remains one of the most deadly diseases in the world [1]. Three major cancer therapies (surgery, chemotherapy, and radiation) have dominated cancer treatment over the last decades. Each method aims to kill or remove cancer while sparing normal tissue. However, each method is well known for causing substantial damage to normal tissues, both within and outside the

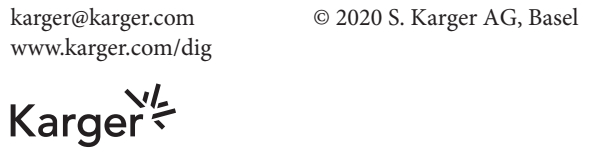

Tadanobu Nagaya

Department of Gastoenterology Shinshu University School of Medicine 3-1-1 Asahi, Matsumoto 390-8621 (Japan) nagaya@shinshu-u.ac.jp 


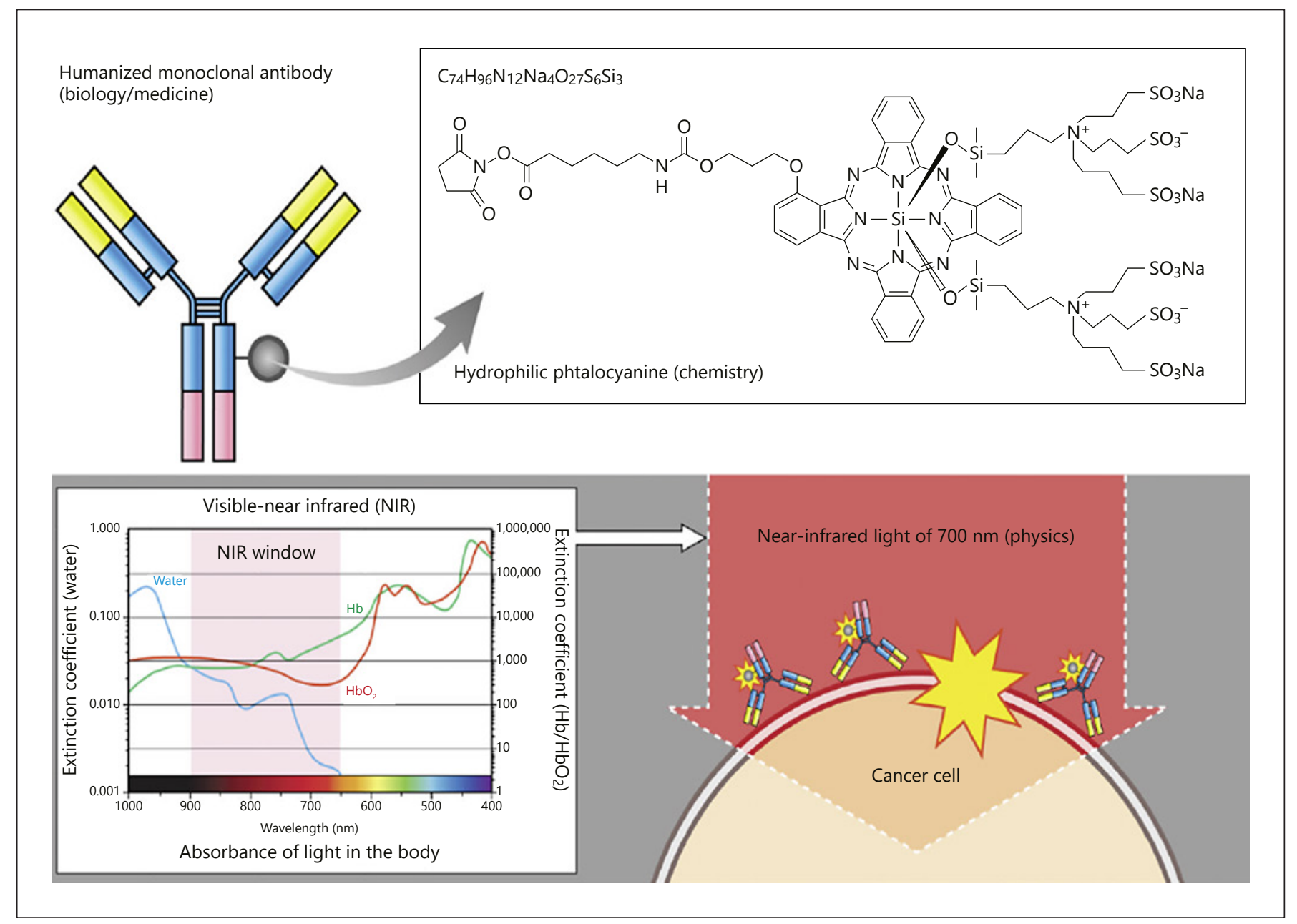

Fig. 1. A schema for explaining selective cancer therapy with NIRPIT. A schema for explaining the photo-physical, chemical, and biological basis of NIR-PIT. Humanized mAbs are employed as targeting moieties because of their high binding specificity. A hydrophilic phthalocyanine dye is employed as an activatable cytotoxic "Nano-dynamite" reagent because of its absorption of near- infrared light of $700 \mathrm{~nm}$ and strong cytotoxicity induced only when bound to the cell membrane. Near-infrared light of $700 \mathrm{~nm}$ is employed as an initiator of cytotoxicity because it is absorbed by IR700 dye and has favorable tissue light penetration. NIR-PIT, near-infrared photoimmunotherapy; ICD, immunogenic cell death. gastrointestinal (GI) tract, leading to adverse events in patients. Among treatments for malignancies arising from the GI tract, the development of antitumor agents has contributed to improved prognosis; however, treatment efficacy and safety remain concerns [2]. New cancer therapies are awaited that improve the outcomes of patients with GI malignancies without causing unacceptable side effects.

Near-infrared photoimmunotheraphy (NIR-PIT) is a newly developed cancer treatment that employs a highly targeted monoclonal antibody conjugated to a photoabsorber [3]. After intravenous injection, each antibodyphotoabsorber conjugate (APC) binds to its cognate re- ceptor overexpressed on the surface of cancer cells. Then, NIR light at $690 \mathrm{~nm}$ is delivered to excite the photo-activatable silica-phthalocyanine dye, IRDye700DX (IR700) on the APC, leading to lethal damage to the cell membrane. Cell death is highly selective with essentially no bystander effect (Fig. 1). NIR-PIT induces a strong and tumor-selective immune response that also has minimal bystander effect. NIR-PIT has been shown to be effective with a variety of different antibodies conjugated to the same IR700 dye [4-10]. A first-in-human phase 1/2 clinical trial of NIR-PIT using cetuximab-IR700 (RM-1929) targeting epidermal growth factor receptor (EGFR) in patients with inoperable head and neck squamous cell can- 
Table 1. Two types of cell death

\begin{tabular}{lll}
\hline & Apoptotic cell death & ICD \\
\hline Immunity & Nonimmunogenic & Immunogenic \\
Cell death & Biological death & Physical/chemical death \\
Time to cell death & $>6$ hours to days & Seconds to minutes \\
Induced by & <onselective $>$ nonselective $>$ & Heat \\
& Drugs/chemotherapy & Cryotherapy \\
& Radiation & Focused ultrasound \\
& Photodynamic therapy & $<$ selective $>$ \\
& <selective & NIR-PIT \\
& Molecular target & \\
Antibodies & \\
& Small molecules & \\
\hline
\end{tabular}

NIR-PIT, near-infrared photoimmunotherapy; ICD, immunogenic cell death.

cer successfully concluded in late 2017 (https://clinicaltrials.gov/ct2/show/NCT02422979). In 2018, a global phase 3 clinical trial began (https://clinicaltrials.gov/ct2/show/ NCT03769506). Results of the phase $1 / 2$ trials suggested NIR-PIT was at least equal if not superior to existing second- and third-line therapies for recurrent head and neck cancers with much fewer adverse events. Thus, NIR-PIT appears to be a promising new type of cancer therapy. In this review article, we focus on a recently developed cancer treatment, NIR-PIT.

\section{Specific Features of Near-Infrared Photoimmunotheraphy}

\section{Highly Specific Cell Killing}

NIR-PIT differs from conventional cancer therapies in its selectivity for killing cancer cells. The specificity derives from targeting by $\mathrm{mAbs}$ which bind to cells overexpressing the cognate antigen. Therefore, nontarget-expressing cells immediately adjacent to targeted cells suffer no toxic effect [3]. Cell killing is initiated by excitation of the APC with nonthermal near-infrared light. NIR light is nonionizing, causes no damage to DNA, and is harmless to nontarget (normal) cells as it is delivered in doses that do not significantly alter tissue temperature. Furthermore, by itself, IR700 is a water-soluble photoreactive dye with no phototoxic or biotoxic properties of its own; therefore, unbound IR700 that dissociates from the APC is readily excreted in urine causing no toxicity $[3,11,12]$. Only under the combination of the target-specific APC and the tumor-targeted exposure of NIR light does this highly targeted cancer therapy result in cellular damage, thus causing minimal to no damage to normal tissues. Indeed, very few side effects have been seen, and many patients have experienced dramatic reductions in tumor size in the clinical trials. Because of its safety profile, NIRPIT also is not limited by the number of treatments that can be administered.

\section{Immunogenic Cell Death}

An important aspect of NIR-PIT is its ability to evoke a strong antitumor immune response. Cells treated with NIR-PIT undergo rapid volume expansion, leading to rupture of the cell membrane and rapid release of cancerspecific antigens. This induces activation of local dendritic cells (DCs), which prime and educate cancer-specific naïve $\mathrm{T}$ cells, leading to proliferation and cell-mediated cancer cell killing $[10,13-15]$. This process is known as immunogenic cell death, in contrast to most other cancer treatments that result in apoptosis (Table 1). After NIRPIT signaling, moieties including calreticulin, ATP, and HMGB1 effectively promote maturation of immature DCs, which are capable of digesting the cancer cell debris [15]. Then, newly primed CD8+ T cells react to a larger repertoire of cancer antigens and proliferate in treated tumor beds [13]. Therefore, anticancer host immunity is strengthened after NIR-PIT largely because of the reeducation and subsequent proliferation of CD8+ T cells. Again, this response is highly tumor specific as it relies on released tumor neoantigens. Thus, NIR-PIT is a highly effective way to induce immunogenic cell death on target cancer cells.

To further enhance the immune response cancer-targeted NIR-PIT could be combined with conventional systemic immunotherapy, which could further activate 


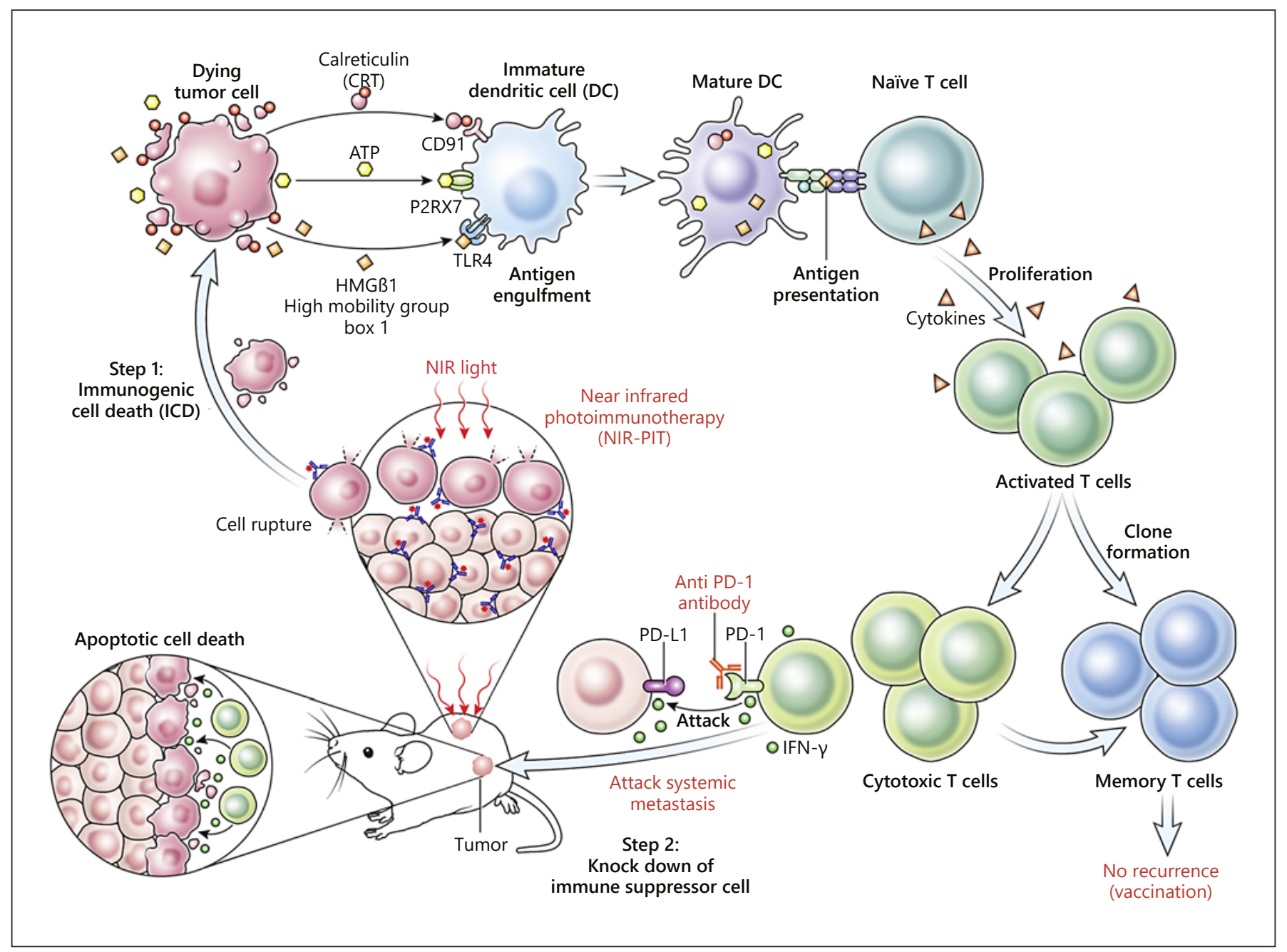

Fig. 2. Proposed mechanism of combination therapy with NIRPIT and systemic PD-1 mAb. Following NIR-PIT cellular damage, tumor cells undergo ICD with release of innate immune ligands, such as CRT, ATP, and HMGB1, along with multiple tumor antigens. Following processing and presentation of these antigens by mature APCs such as DCs, a systemic polyclonal T cell response develops. However, PD-1 expression on activated CD8+ T cells and PD-L1 expression within the tumor microenvironment leads to adaptive immune resistance and dysfunctional T cells. PD-1

CD8+ T cells after NIR-PIT. Animal models that received combination therapy with NIR-PIT and immune-checkpoint inhibitors demonstrated immediate tumor shrinkage within several days and cures in many animals. Not only did the treated tumor respond but metastases also shrank and disappeared within several days suggesting an abscopal response. Furthermore, the animals that experience complete response rejected any attempts to reinoculate the tumor, suggesting that these mice had gained immunity against the initial tumor (Fig. 2) [13]. Similarly,
$\mathrm{mAb}$ reverses this adaptive immune resistance resulting in activation of tumor-infiltrating lymphocytes specific for multiple tumor antigens at both primary and distant (abscopal) sites of disease. This leads to complete tumor rejection and immunologic memory. NIR-PIT, near-infrared photoimmunotherapy; DC, dendritic cell; ICD, immunogenic cell death; PD-L1, programmed death-ligand 1; $\mathrm{mAb}$, monoclonal antibody; APC, antibody-photoabsorber conjugate; CRT, calreticulin; HMGB, high mobility group box.

NIR-PIT can be directed at immunomodulatory cells such as Tregs and myeloid-derived suppressor cells leading to immediate reductions of immunosuppressive cells in the tumor microenvironment. Thus, NIR-PIT and some form of adjuvant immunotherapy might be the ideal future direction for NIR-PIT.

\section{Super-Enhanced Permeability and Retention Effects}

Another unique feature of NIR-PIT is its immediate effect on tumor vascularity. Some degree of enhanced 


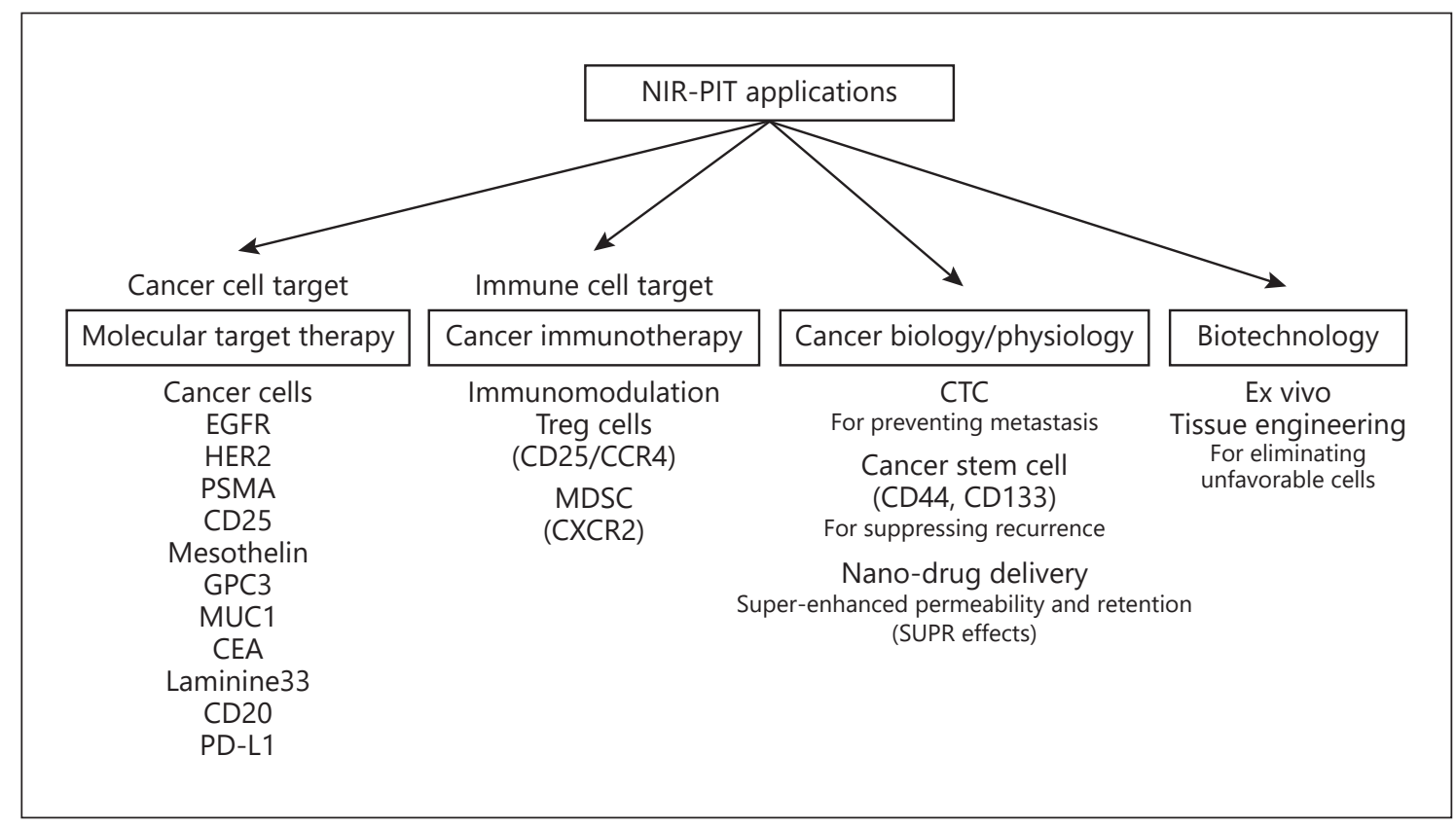

Fig. 3. NIR-PIT applications. NIR-PIT can be applied to many cell types that overexpress specific target membrane antigens for which there is a suitable mAb. NIR-PIT, near-infrared photoimmunotherapy; mAb, monoclonal antibody.

permeability and retention (EPR) is present in most tumors due to vascular leakiness. In contrast, rapid and massive cancer cell killing adjacent to tumor vessels after NIR-PIT leads to an immediate and dramatic increase in vascular permeability after therapy [16-19]. The delivery of various nano-sized or macromolecular drugs into an NIR-PIT treated tumor increases up to 24-fold compared with that in a control nontreated tumor immediately after treatment, a phenomenon that is known as the super-enhanced permeability and retention (SUPR) which is multifold more pronounced than EPR $[16,18]$. By inducing immediate necrosis in the perivascular cancer cells, a space forms between the vessels and the remaining tumor, allowing the vessel to enlarge, increasing blood volume, and decreasing blood velocity. Consequently, improved delivery of nano-sized therapeutic agents into the treated tissue occurs. Therefore, a combination therapy using NIR-PIT followed by a nano-sized anticancer agent could be more effective than either therapy alone $[4,18]$. SUPR effects also allow for enhanced delivery for other antibodies and APCs, whose intra-tumoral distribution is enhanced by increased leakage into the tumor bed after NIR-PIT [20-22].

\section{Applications of Near-Infrared Photoimmunotherapy}

NIR-PIT can be applied to any cancer with an overexpressed target membrane antigen for which there is a suitable humanized mAb (Fig. 3). NIR-PIT has been successfully performed with APCs targeting many antigens, such as EGFR in the case of skin cancer [23], lung cancer [24], and breast cancer [11], human epidermal growth factor receptor 2 in the case of gastric cancer [25, 26], prostatespecific membrane antigen in the case of prostate cancer [27], mesothelin in the case of mesothelioma, pancreatic, and ovarian cancer [9], Glypican 3 (GPC3) in the case of hepatocellular carcinoma [4], and CD20 in the case of malignant lymphoma [8]. Furthermore, NIR-PIT targeting programmed death-ligand 1 (PD-L1) for PD-L1 expressing cancer cells [28], CD25 for regulatory $\mathrm{T}$ cells [10], and CD44 for cancer stem cells has also proven successful [5].

Circulating tumor cells are 1 mechanism by which tumors can metastasize. Circulating tumor cells are known to harbor characteristic cell surface markers that may be targeted by NIR-PIT with specific APCs in future.

NIR-PIT may also have applications in tissue engineering. The new field of tissue engineering allows stem cells to be placed on specific scaffolds to grow new organs or heal wounds. Unfortunately, during the growth of 
these cells, teratomas, representing aberrant differentiation of pluripotent stem cells, may develop, rendering the graft useless. NIR-PIT could be used to eliminate teratomas from the $2 \mathrm{D}$ - or $3 \mathrm{D}$-graft without damaging the other cells and thus save the graft from being discarded [29, 30] (Fig. 3).

\section{Near-Infrared Photoimmunotherapy for Gastrointestinal Tract Cancers}

The therapeutic effects of NIR-PIT depend on access of the APC to the target cancer cells and the ability to deliver NIR light to that tumor. NIR light can be easily delivered to lesions near the surface such as in the mouth and skin by means of an external light source. Theoretically, NIR light can penetrate up to $2 \mathrm{~cm}$ into tissue [31]. However, in more deeply located tumors such as GI tract cancer, light is rapidly attenuated in tissue, and therefore, the light source must originate either from within the tumor or nearby. There are several potential solutions to this problem. For instance, NIR light could be delivered to a tumor during open or laparoscopic surgical resection, thus treating residual tumor. Alternatively, light could be administered endoscopically as in the case of esophageal, stomach, and colorectal cancer. A third possibility is to introduce light fibers interstitially into tumors using the techniques of endoscopic ultrasound-guided fine-needle aspiration for targeting pancreatic cancer and peritoneally disseminated cancer.

We have shown that a fiber optic light diffuser suitable for NIR can be placed within or near tumors during endoscopy to effectively treat peritoneally disseminated gastric cancer with NIR-PIT in a mouse model. NIR-PITtreated tumors by endoscopy showed necrosis and microhemorrhage with scattered clusters of remaining damaged cancer cells; however, cancer cells without damage were observed in the control tumor (Fig. 4) [25]. Such procedures could be performed more easily in human patients than in mouse models because of size constraints in the mouse, and endoscopy can be conducted with better flexibility in humans. Additionally, compared to other cancer therapies, NIR light administered via endoscopic optical fiber diffuser is a relatively minimally invasive procedure. The ability to deliver light endoscopically makes NIR-PIT particularly attractive for GI tract malignancies. Applications in esophageal, gastric, and duodenal malignancies using such antibodies as anti-EGFR and anti-HER2 are clear possibilities. Anal and colorectal cancers using anti-

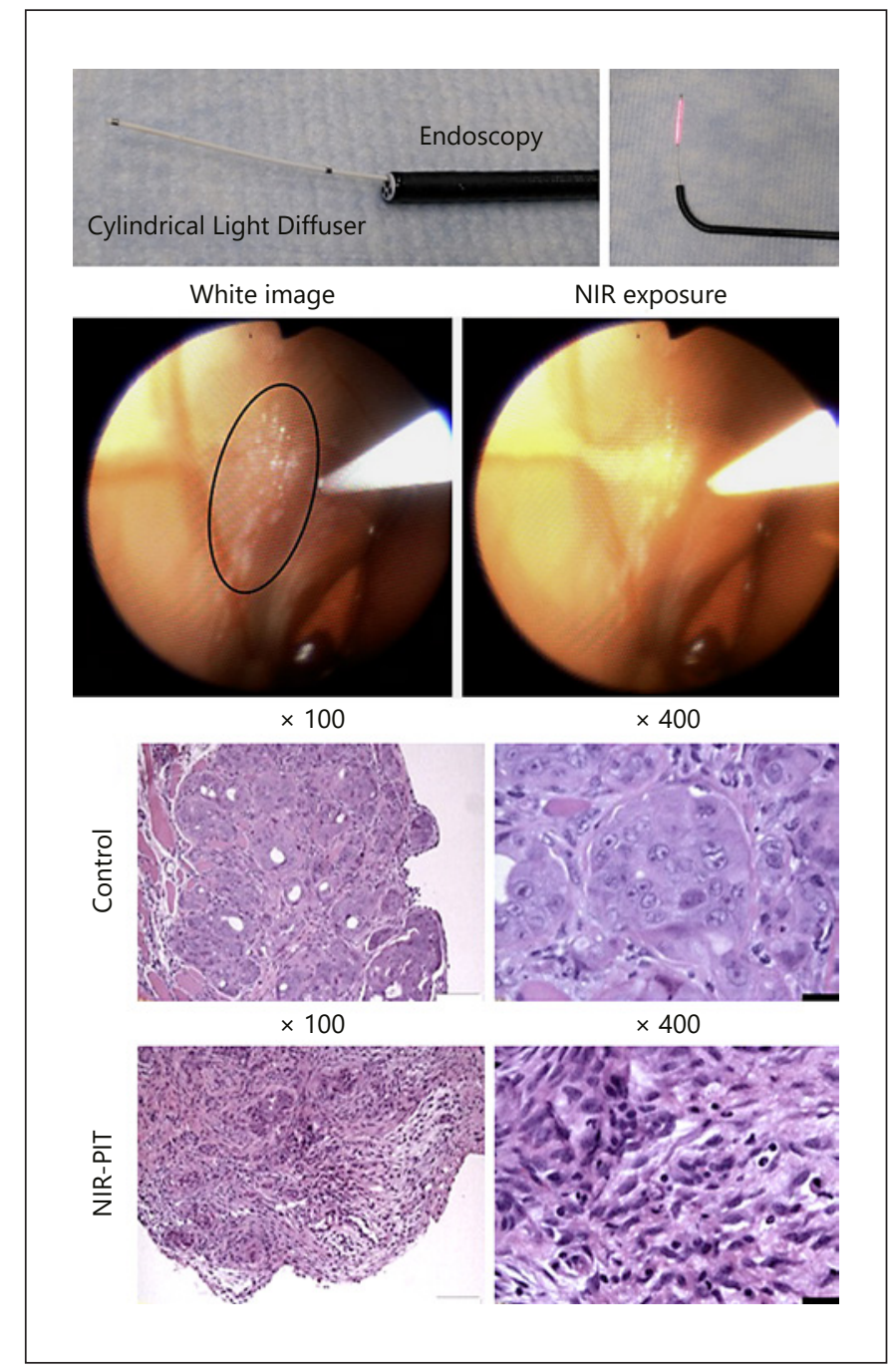

Fig. 4. Endoscopic NIR-PIT. A cylindrical light diffuser can be used in clinical use endoscopy (upper panel). In vivo peritoneally disseminated gastric cancer (human gastric cancer cell line; N87 cell) in a mouse model (white small nodules in black circle) can be exposed to NIR light using the cylindrical light diffuser through the endoscopy and the distance of light can be easily adjusted (middle panel). Histological specimens of disseminated gastric cancers are shown. All specimens are stained with Hematoxylin and Eosin. No obvious damage was observed in control tumor (left). NIR-PIT treated tumors show necrosis and micro-hemorrhage with scattered clusters of remaining damaged cancer cells (right). White bar is $100 \mu \mathrm{m}$, and black bar is $20 \mu \mathrm{m}$ (lower panel). NIR-PIT, nearinfrared photoimmunotherapy.

EGFR and other antibodies are potential target tumors. Hepatocellular (anti-GCP3) and pancreatic (anti-mesothelin, and anti-FAP) as well as peritoneal metastases from GI malignancies are also possible applications for NIR-PIT. 


\section{Conclusion}

NIR-PIT has great potential to become a widely applicable new type of cancer therapy. When combined with immune-activating therapies, NIR-PIT not only treats the targeted local tumor but also reduces or eliminates systemic metastasis and prevents recurrence in some animal models. In clinical settings, NIR light could be delivered using endoscopes to accommodate a number of anatomic sites throughout the body in which it is possible to insert endoscopic devices. Alternatively, intraoperative or interstitial light delivery could be used in GI malignancies not amenable to endoscopic NIR-PIT. Thus, NIRPIT could be a promising candidate for treating GI tract cancers.

\section{Conflict of Interest Statement}

No potential conflicts of interest were disclosed.

\section{Funding Sources}

This research was supported by the Intramural Research Program of the US National Institutes of Health, National Cancer Institute, Center for Cancer Research (ZIA BC011513).

\section{Author Contributions}

Conception and design: T. Nagaya, P.L. Choyke, and H. Kobayashi. Writing, review, and/or revision of the manuscript: $\mathrm{T}$. Nagaya, P.L. Choyke, and H. Kobayashi.

\section{References}

1 Torre LA, Siegel RL, Ward EM, Jemal A. Global cancer incidence and mortality rates and trends: an update. Cancer Epidemiol Biomarkers Prev. 2016;25(1):16-27.

2 Watanabe M, Baba H, Ishioka C, Nishimura $\mathrm{Y}$, Muto M. Recent advances in diagnosis and treatment for malignancies of the gastrointestinal tract. Digestion. 2012;85(2):95-8.

3 Mitsunaga M, Ogawa M, Kosaka N, Rosenblum LT, Choyke PL, Kobayashi H. Cancer cell-selective in vivo near infrared photoimmunotherapy targeting specific membrane molecules. Nat Med. 2011;17(12):1685-91.

4 Hanaoka H, Nagaya T, Sato K, Nakamura Y, Watanabe R, Harada T, et al. Glypican-3 targeted human heavy chain antibody as a drug carrier for hepatocellular carcinoma therapy. Mol Pharm. 2015;12(6):2151-7.

5 Nagaya T, Nakamura Y, Okuyama S, Ogata F, Maruoka Y, Choyke PL, et al. Syngeneic mouse models of oral cancer are effectively targeted by anti-CD44-based NIR-PIT. Mol Cancer Res. 2017;15:1667-77.

6 Nani RR, Gorka AP, Nagaya T, Yamamoto T, Ivanic J, Kobayashi $\mathrm{H}$, et al. Activation of duocarmycin-antibody conjugates by near-infrared light. ACS Cent Sci. 2017;3(4):329-37.

7 Nakamura Y, Mochida A, Nagaya T, Okuyama S, Ogata F, Choyke PL, et al. A topicallysprayable, activatable fluorescent and retaining probe, SPiDER-betaGal for detecting cancer: advantages of anchoring to cellular proteins after activation. Oncotarget. 2017;8: 39512-21.

8 Nagaya T, Nakamura Y, Sato K, Harada T, Choyke PL, Kobayashi H. Near infrared photoimmunotherapy of B-cell lymphoma. Mol Oncol. 2016;10(9):1404-14.

9 Nagaya T, Nakamura Y, Sato K, Zhang YF, Ni M, Choyke PL, et al. Near infrared photoimmunotherapy with an anti-mesothelin antibody. Oncotarget. 2016;7(17):23361-9.
10 Sato K, Sato N, Xu B, Nakamura Y, Nagaya T, Choyke PL, et al. Spatially selective depletion of tumor-associated regulatory $\mathrm{T}$ cells with near-infrared photoimmunotherapy. Sci Transl Med. 2016;8(352):352ra110.

11 Nagaya T, Sato K, Harada T, Nakamura Y, Choyke PL, Kobayashi H. Near infrared photoimmunotherapy targeting EGFR positive triple negative breast cancer: optimizing the conjugate-light regimen. PLoS One. 2015; 10(8):e0136829.

12 Okuyama S, Nagaya T, Ogata F, Maruoka Y, Sato K, Nakamura Y, et al. Avoiding thermal injury during near-infrared photoimmunotherapy (NIR-PIT): the importance of NIR light power density. Oncotarget. 2017;8(68): 113194-201.

13 Nagaya T, Friedman J, Maruoka Y, Ogata F, Okuyama S, Clavijo PE, et al. Host immunity following near-infrared photoimmunotherapy is enhanced with PD-1 checkpoint blockade to eradicate established antigenic tumors. Cancer Immunol Res. 2019;7(3): 401-13.

14 Nakamura Y, Nagaya T, Sato K, Harada T, Okuyama S, Choyke PL, et al. Alterations of filopodia by near infrared photoimmunotherapy: evaluation with 3D low-coherent quantitative phase microscopy. Biomed Opt Express. 2016;7(7):2738-48.

15 Ogawa M, Tomita Y, Nakamura Y, Lee MJ, Lee S, Tomita S, et al. Immunogenic cancer cell death selectively induced by near infrared photoimmunotherapy initiates host tumor immunity. Oncotarget. 2017;8(6): 10425-36.

16 Kobayashi H, Choyke PL. Super enhanced permeability and retention (SUPR) effects in tumors following near infrared photoimmunotherapy. Nanoscale. 2016;8(25):12504-9.
17 Kobayashi H, Watanabe R, Choyke PL. Improving conventional enhanced permeability and retention (EPR) effects; what is the appropriate target? Theranostics. 2013;4(1):819.

18 Sano K, Nakajima T, Choyke PL, Kobayashi $\mathrm{H}$. Markedly enhanced permeability and retention effects induced by photo-immunotherapy of tumors. ACS Nano. 2013;7(1): $717-24$

19 Sano K, Nakajima T, Choyke PL, Kobayashi $\mathrm{H}$. The effect of photoimmunotherapy followed by liposomal daunorubicin in a mixed tumor model: a demonstration of the superenhanced permeability and retention effect after photoimmunotherapy. Mol Cancer Ther. 2014;13(2):426-32.

20 Liang CP, Nakajima T, Watanabe R, Sato K, Choyke PL, Chen Y, et al. Real-time monitoring of hemodynamic changes in tumor vessels during photoimmunotherapy using optical coherence tomography. J Biomed Opt. 2014; 19(9):98004.

21 Tang Q, Nagaya T, Liu Y, Horng H, Lin J, Sato $\mathrm{K}$, et al. 3D mesoscopic fluorescence tomography for imaging micro-distribution of antibody-photon absorber conjugates during near infrared photoimmunotherapy in vivo. J Control Release. 2018;279:171-80.

22 Tang Q, Nagaya T, Liu Y, Lin J, Sato K, Kobayashi $\mathrm{H}$, et al. Real-time monitoring of microdistribution of antibody-photon absorber conjugates during photoimmunotherapy in vivo. J Control Release. 2017;260: 154-63.

23 Mitsunaga M, Nakajima T, Sano K, Choyke PL, Kobayashi H. Near-infrared theranostic photoimmunotherapy (PIT): repeated exposure of light enhances the effect of immunoconjugate. Bioconjug Chem. 2012;23(3): 604-9. 
24 Sato K, Nagaya T, Mitsunaga M, Choyke PL, Kobayashi H. Near infrared photoimmunotherapy for lung metastases. Cancer Lett. 2015;365(1):112-21.

25 Nagaya T, Okuyama S, Ogata F, Maruoka Y, Choyke PL, Kobayashi H. Endoscopic near infrared photoimmunotherapy using a fiber optic diffuser for peritoneal dissemination of gastric cancer. Cancer Sci. 2018;109(6):19028.

26 Nagaya T, Okuyama S, Ogata F, Maruoka Y, Choyke PL, Kobayashi H. Near infrared photoimmunotherapy using a fiber optic diffuser for treating peritoneal gastric cancer dissemination. Gastric Cancer. 2019;22(3):463-72.
27 Nagaya T, Nakamura Y, Okuyama S, Ogata F, Maruoka Y, Choyke PL, et al. Near-infrared photoimmunotherapy targeting prostate cancer with prostate-specific membrane antigen (PSMA) antibody. Mol Cancer Res. 2017; 15(9):1153-62.

28 Nagaya T, Nakamura Y, Sato K, Harada T, Choyke PL, Hodge JW, et al. Near infrared photoimmunotherapy with avelumab, an anti-programmed death-ligand 1 (PD-L1) antibody. Oncotarget. 2017;8(5):8807-17.
29 Sato K, Choyke PL, Hisataka K. Selective cell elimination from mixed 3D culture using a near infrared photoimmunotherapy technique. J Vis Exp. 2016(109):53633.

30 Sato K, Nakajima T, Choyke PL, Kobayashi H. Selective cell elimination in vitro and in vivo from tissues and tumors using antibodies conjugated with a near infrared phthalocyanine. RSC Adv. 2015;5(32):25105-14.

31 Henderson TA, Morries LD. Near-infrared photonic energy penetration: can infrared phototherapy effectively reach the human brain? Neuropsychiatr Dis Treat. 2015;11: 2191-208. 